\title{
Frank rectal bleeding: a prospective study of causes in patients over the age of 40
}

\author{
P.S.Y. Cheung, S.K.C. Wong, J. Boey and C.K. Lai \\ Department of Surgery, University of Hong Kong, Queen Mary Hospital, Hong Kong
}

\begin{abstract}
Summary: In order to determine the frequency of tumours in patients presenting with frank rectal bleeding, a prospective study was carried out in 337 patients over the age of 40 attending our Proctology Clinic. After making a clinical diagnosis, flexible sigmoidoscopy followed by barium enema was performed. Excluding 7 digitally palpable rectal cancers, there were 30 cancers (9.1\%), 34 polyps (10.3\%), 7 proctocolitis $(2.1 \%)$ and 25 diverticula $(7.6 \%)$ detected, giving a total diagnostic yield of $29.1 \%$. A clinical diagnosis of bleeding sources other than haemorrhoids was made in $80 \%$ of the cancers. Flexible sigmoidoscopy detected $93.3 \%$ of the cancers and $88.2 \%$ of the polyps. Barium enema diagnosed the remaining cancers and polyps but there was a false-positive rate of 3.8\% . From our results, we conclude that patients with frank rectal bleeding should be screened routinely for left-colon cancer irrespective of the clinical diagnosis. The flexible sigmoidoscope is a quick and useful tool. However, barium enema should be recommended to individuals with strong clinical suspicion of rectal bleeding other than haemorrhoids.
\end{abstract}

\section{Introduction}

Colorectal cancer is one of the commonest internal malignancies in both sexes. As the incidence of colorectal cancer rises after the age of $40,{ }^{1}$ programmes have been designed for screening asymptomatic patients with the hope of achieving an earlier diagnosis. The problem of occult bleeding and the effectiveness of the stool Haemoccult test as a screening procedure have been widely discussed. $^{2,3}$ Yet in clinical proctological practice, clinicians are often uncertain about the extent of investigation necessary for patients presenting with frank rectal bleeding.

Haemorrhoids are by far the commonest cause of frank rectal bleeding. As colorectal cancer, especially left-sided lesion, often manifests as frank rectal bleeding, it can easily be overlooked when haemorrhoids coexist, unless concurrent colonic symptoms such as altered bowel habits or weight loss are also present.

Anal fissure, although it also gives rise to frank rectal bleeding, often presents with painful bowel movement. The diagnosis is obvious from the history together with the presence of an anal ulcer. Investigation has to be delayed until anal pain subsides and by that time, bleeding usually subsides and does not pose further problems.

Correspondence: P.S.Y. Cheung F.R.C.S.(Glasg)

Accepted: 22 December 1987
The purpose of this study was to evaluate prospectively the accuracy of clinical judgement in managing patients presenting with frank rectal bleeding, and to estimate the tumour yield of flexible sigmoidoscopy and barium enema employed as diagnostic modalities.

\section{Patients and methods}

Three hundred and thirty-seven consecutive patients over the age of $\mathbf{4 0}$ who presented to our Proctology Clinic in a two-year period with the major complaint of frank rectal bleeding were included in the study. There were 182 men and 155 women. Their mean age was 58.9 years with a range of 40 to 93 years. They were usually referred from general practitioners with the diagnosis of 'bleeding piles' or 'per rectum bleeding'. Those referred with an obvious diagnosis of malignancy or massive rectal bleeding were excluded from the study. Patients with anal fissures were excluded from the study as they usually present with painful bowel movement.

The management scheme included clinical examination followed by flexible sigmoidoscopy, and then barium enema. In the clinical history, the following points were sought: length of bleeding,

(C) The Fellowship of Postgraduate Medicine, 1988 
colour of blood, whether the blood was mixed in faecal matter, passage of mucus, presence of tenesmus, change in bowel habits, recent weight loss and abdominal pain. The presence of anaemia or an abdominal mass was looked for in the physical examination. A digital rectal examination was performed to detect any palpable rectal lesions. Proctoscopy was performed and haemorrhoids present were injected with $5 \%$ phenol in almond oil or band-ligated at the same setting. A clinical diagnosis of non-haemorrhoidal pathology was made when there was presence of altered blood mixed with stool, or the presence of mucus, tenesmus, altered bowel habit, weight loss, significant abdominal pain, abdominal or rectal masses. Patients with digitally palpable rectal cancers were excluded from further investigation.

The patients were then scheduled for flexible sigmoidoscopic examination on the day after their first visit. The bowel was prepared at home using two doses of $30 \mathrm{ml} 50 \%$ magnesium sulphate in the evening before and on the morning of the examination. A Fleet enema was also given on the day of examination. No premedication was used. The procedure was performed by colorectal surgeons using a $110 \mathrm{~cm}$ Olympus IBW flexible sigmoidoscope but limiting scope insertion to the $60 \mathrm{~cm}$ level only, so as to simulate a $60 \mathrm{~cm}$ flexible sigmoidoscope. The extent of examination was determined by radiographic screening. Significant pathology including tumours, diverticula or proctocolitis were noted and relevant lesions biopsied. Polyps were removed by snare polypectomy.

A barium enema was booked for all patients on their first visit. Any defaulters were contacted and their rectal bleeding history was inquired. Barium enema was omitted when the presence of cancer was confirmed by sigmoidoscopic biopsy.

The yield of cancer and other significant pathology in the patients under study were analysed at various steps along the management scheme.

\section{Results}

\section{Diagnostic yield}

On clinical grounds alone, 250 patients were diagnosed to have haemorrhoidal bleeding while 87 were suspected to have other causes of bleeding. Of these 87 patients, 31 were found to have colorectal cancers ( 7 had digitally palpable rectal cancers), 9 had colonic polyps, 7 had proctocolitis and 7 patients had diverticular disease. Nineteen patients had normal findings in both flexible sigmoidoscopy and barium enema and 14 patients had only flexible sigmoidoscopy examinations which were normal. The seven patients with digitally palpable rectal cancers were excluded from further statistical evaluation of data.

Of the 250 patients diagnosed clinically to have haemorrhoids alone, 6 were found to have cancer, 25 had colonic polyps, 1 had proctocolitis and 18 had diverticular disease; 124 patients had normal colonic examination and 76 patients had limited colon examination by flexible sigmoidoscopy only which were normal (Table I).

Three hundred and twenty-seven patients underwent flexible sigmoidoscopic examination and 212 patients underwent barium enema examination. The diagnostic yield by flexible sigmoidoscopy and barium enema is shown in Table II. Flexible sigmoidoscopy detected 28 cancers, and had a total diagnostic yield of $20.5 \%$ for significant pathology. The remaining examinations were normal.

Among the 212 barium enema examinations, the pathology detected included 2 right-sided colonic cancers, 4 polyps, and 25 diverticular disease. Eight examinations showed suspicious narrowing in the colon: 1 in the rectosigmoid, 4 in the sigmoid, 1 in the ascending colon and 2 in the caecum. These all subsequently proved to be normal by colonoscopy giving a false positive rate of $3.8 \%$.

\section{Site of lesions}

The distribution of cancers is shown in Figure 1. All 28 left-sided colonic cancers were detected by flexible sigmoidoscopy. Their presence was suspected clinically in 24 patients, the other 4 were

Table I Outcome of 337 patients with frank rectal bleeding

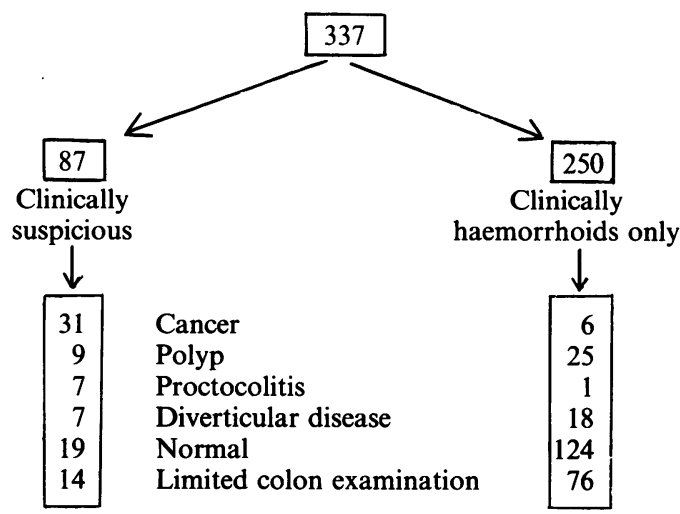


Table II Diagnostic yield of flexible sigmoidoscopy and barium enema in 330 patients with frank rectal bleeding (7 patients with digitally palpable cancer were excluded).

\begin{tabular}{|c|c|c|c|c|}
\hline & \multicolumn{2}{|c|}{$\begin{array}{c}\text { Flexible } \\
\text { sigmoidoscopy }\end{array}$} & \multicolumn{2}{|c|}{$\begin{array}{l}\text { Barium } \\
\text { enema }\end{array}$} \\
\hline & $n$ & $(\%)$ & $n$ & $(\%)$ \\
\hline \multicolumn{5}{|l|}{ Study done } \\
\hline Cancer & 28 & (8.6) & 2 & $(0.9)$ \\
\hline Polyp & 30 & $(9.2)$ & 4 & (1.9) \\
\hline Proctocolitis & 8 & (2.4) & 0 & \\
\hline Diverticula & 1 & $(0.3)$ & 25 & (11.8) \\
\hline Suspicious narrowing & 0 & & 8 & $(3.8)$ \\
\hline \multirow[t]{2}{*}{ Normal } & 260 & $(79.5)$ & 173 & (81.6) \\
\hline & 327 & & 212 & \\
\hline \multicolumn{5}{|l|}{ Study not done } \\
\hline Cancer & - & & $28^{*}$ & \\
\hline Asymptomatic & - & & 58 & \\
\hline \multirow[t]{2}{*}{ Defaulted } & 3 & & 32 & \\
\hline & 3 & & 118 & \\
\hline Total & 330 & & 330 & \\
\hline
\end{tabular}

*Barium enema omitted when diagnosis of cancer confirmed.

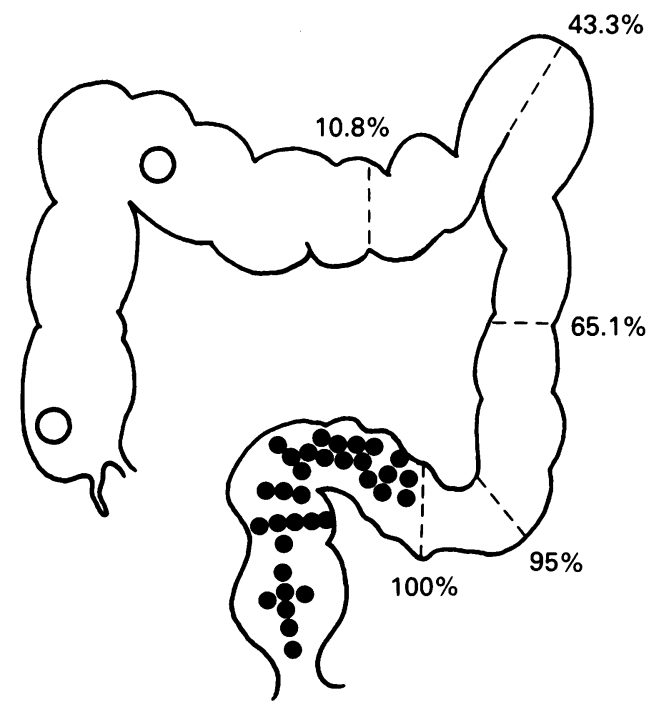

Figure 1 Cancer distribution $(n=37)$ and the percentage of sigmoidoscopic examinations reaching the indicated level of colon at $60 \mathrm{~cm}$ as determined by radiographic screening. Polyps detected by sigmoidoscopy $(\Theta)$ or barium enema $(O)$. thought to have haemorrhoids only. Twelve cancers were situated above the $20 \mathrm{~cm}$ level by flexible sigmoidoscopy, and 16 were either at or below the $20 \mathrm{~cm}$ level.

The two right-sided colonic cancers were beyond the reach of the sigmoidoscope (Figure 1). Both patients were clinically diagnosed to have haemorrhoids only. The patient with a hepatic flexure tumour actually presented with anaemia to the physician, who discovered bleeding haemorrhoids and referred the patient after blood transfusion. The other patient had a caecal tumour with coexisting bleeding haemorrhoids. The clinical diagnosis of the cause of rectal bleeding was correct, and the caecal tumour was asymptomatic at referral. However, when the barium enema examination was performed 3 months later, obvious symptoms of colonic malignancy including alternating constipation and diarrhoea, abdominal pain and weight loss appeared.

The distribution of polyps is shown in Figure 2. Most of the polyps were located in the rectum and sigmoid colon. Thirty-one of the 34 polyps were removed by snare polypectomy. Three patients who had polyps in the transverse colon or descending colon detected by barium enema refused polypectomy because their rectal bleeding ceased after haemorrhoid treatment. Twenty-six of the 318 polyps removed were retrieved for histological section. The remaining 5 polyps were small (2-6) $5 \mathrm{~mm}$ ) and were lost during withdrawal of the sigmoidoscope and could not be recovered in the

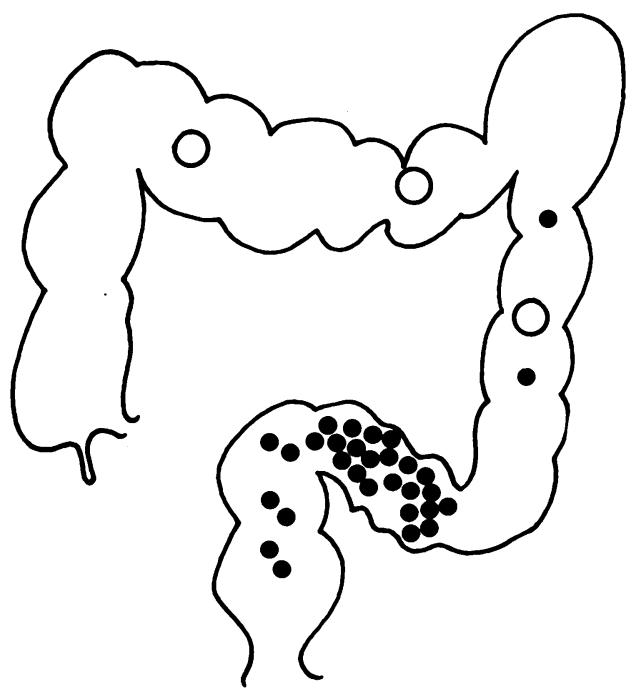

Figure 2 Distribution of polyps $(n=34)$. Cancer site detected by sigmoidoscopy $(O)$ or barium enema $(O)$. 
stool. The pathology of the removed polyps showed 18 tubular adenoma, 4 villotubular adenoma, 1 villous adenoma, 1 carcinoma-in-situ in a $1 \mathrm{~cm}$ tubular adenoma and 2 juvenile polyps. The median size of the polyp was $5 \mathrm{~mm}$ with a range of 2 to $20 \mathrm{~mm}$. Two were greater than $1 \mathrm{~cm}$ and both were juvenile polyps. Eight were $1 \mathrm{~cm}$, and 24 were smaller than $1 \mathrm{~cm}$ in size.

\section{Discussion}

Our study revealed an overall cancer yield of $10.8 \%$ (37 of 337) among patients over the age of 40 presenting with frank rectal bleeding. Excluding the 7 digitally palpable rectal cancers, the yield was $9.1 \%$. This figure is relatively high in comparison to the $4.2 \%$ incidence found by Williams and Thomson, ${ }^{4}$ and the $4.6 \%$ shown by Dehn and McGinn. ${ }^{5}$ In both series, the authors included patients of all ages. As haemorrhoidal bleeding occurs commonly in young people, this lack of selection tends to dilute the incidence of cancers detected. We chose to confine our study to patients over the age of 40 because beyond this age the incidence of colorectal cancer rises two-fold every decade. ${ }^{6}$ Meyer et al. ${ }^{7}$ also had a lower cancer yield of $3.2 \%$ in patients above the age of 40 . However, asymptomatic patients and those with a personal or a family history of colorectal cancer or polyp were included. These also reduce the cancer yield.

Our study emphasizes that a detailed history is important because $80 \%(24 / 30)$ of the cancers were suspected clinically. The fact that haemorrhoids often coexist with underlying malignancy should be stressed. In our study, coexisting haemorrhoids were found in two-thirds of the cancers. In the study by Shinya et al. ${ }^{8} 86 \%$ of 1604 patients with bleeding colonic pathology had coexisting haemorrhoids. The finding of seven palpable rectal cancers indicates the importance of careful digital rectal examination which would render diagnosis of malignancy obvious. On the other hand, clinical history alone is not absolutely reliable as six cancers were diagnosed as haemorrhoids only in this prospective study. Routine screening for malignancy is thus advisable in older patients with rectal bleeding.

Flexible sigmoidoscopy is a well established screening procedure for the left colon. Its advantage over rigid sigmoidoscopy has been

\section{References}

1. Winawer, S.J., Sherlock, P., Schottenfeld, D. \& Miller, D.G. Screening for colon cancer. Gastroenterology 1976, 70: 783-789. widely discussed in terms of the extent of examination and the diagnostic yield for colonic pathology.${ }^{9-12}$ In our study, flexible sigmoidoscopy with an insertion length to $60 \mathrm{~cm}$ reached the descending - sigmoid junction in $95 \%$ of cases; $43.3 \%$ could even reach the splenic flexure and $10.8 \%$ reached the distal transverse colon (Figure 1). It detected all of the rectal and sigmoid cancers found in our series. On the other hand, rigid sigmoidoscopy with an average insertion length of $20 \mathrm{~cm}^{10-12}$ can only detect $57.1 \%$ (16 of 28 ) of the left-colon cancers. Our finding favours the use of flexible sigmoidoscopy as a routine screening procedure.

Right-sided colonic cancer generally presents with positive faecal occult blood and anaemia. Of the two patients in our series, one had overt symptoms of anaemia and the other had altered bowel habits and abdominal pain. Both patients had frank rectal bleeding due to coexisting haemorrhoids.

Barium enema has been recommended by some authors ${ }^{13,14}$ as routine screening in rectal bleeding but deemed to be of no value by others. ${ }^{15}$ From our experience, apart from clinical indication for investigating the whole colon, barium enema detected no additional cancer after sigmoidoscopic screening. It also carried a $3.8 \%$ false-positive rate which led to unnecessary colonoscopy. In our opinion, barium enema examination should be recommended only to those with clinical suspicion of a bleeding source other than haemorrhoids.

The detection of polyps during colonic screening has been a subject of interest because of the risk of subsequent cancer formation. ${ }^{16,17}$ Spencer et al. ${ }^{18}$ showed that there was no increased risk of cancer developing in patients with polyps followed up for as long as 20 years. A detailed clinico-pathological study by Muto et al. ${ }^{19}$ showed that the polypcancer sequence had a slow evolution and the majority of adenomas did not become malignant during adult life. Since polyps are detected in between $10 \%$ and $13 \%$ of asymptomatic individuals during routine screening, ${ }^{7,16}$ our finding of $10.3 \%$ polyps, most of which were below $1 \mathrm{~cm}$ in size, is likely to be an incidental finding.

In conclusion, patients with frank rectal bleeding should be screened for the presence of left-colon cancer irrespective of the existence of haemorrhoids. The flexible sigmoidoscope is a useful and convenient tool. However, barium enema should be recommended to individuals with strong clinical suspicion of rectal bleeding not due to haemorrhoids.

2. Sontag, S.J., Durczak, C., Aranha, G.V., Chejfec, G., Frederick, W. \& Greenlee, H.B. Fecal occult blood screening for colorectal cancer in a Veterans 
Administration Hospital. Am J Surg 1983, 145: 89-94.

3. Editorials: Sensitivity of fecal occult blood testing for adenomas. Gastroenterology 1982, 83: 1136-1138.

4. Williams, J.T. \& Thomson, J.P.S. Ano-rectal bleeding: a study of causes and investigative yields. The Practitioner 1977, 219: 327-331.

5. Dehn, T. \& McGinn, F.P. Causes of ano-rectal bleeding. Postgraduate Med J 1982, 58: 92-93.

6. Schottenfeld, D. \& Winawer, S.J. Large intestine. In: Schottenfeld, D. and Fraumeni, J.F. (eds) Cancer Epidemiology and Prevention. W.B. Saunders, Philadelphia, 1982, pp 703-727.

7. Meyer, C.T., McBride, W., Goldblatt, R.S., Borak, J., Marignani, P., Black, H.R. \& McCallum, R.W. Clinical experience with flexible sigmoidoscopy in asymptomatic and symptomatic patients. Yale J Biol Med 1980, 53: 345-352.

8. Shinya, H., Cwern, M. \& Wolf, G. Colonoscopic diagnosis and management of rectal bleeding. Surg Clin N Am 1982, 62: 897-903.

9. Hilsabeck, J.R. Experience with routine office sigmoidoscopy using the $60-\mathrm{cm}$ flexible colonoscope in private practice. Dis Colon Rectum 1983, 26: 314-318.

10. Traul, D.G., Davis, C.B., Pollock, J.C. \& Scudamore, H.H. Flexible fiberoptic sigmoidoscopy - The Monroe Clinic experience. A prospective study of 5000 examinations. Dis Colon Rectum 1983, 26: 161-166.

11. Winnan, G., Berci, G., Panish, J., Talbot, T.M., Overholt, B.F. \& McCallum, R.W. Superiority of the flexible to the rigid sigmoidoscope in routine proctosigmoidoscopy. N Engl J Med 1980, 302: 1011-1012.
12. Marks, G., Gathright, J.B., Boggs, H.W., Ray, J.E., Castro, A.F. \& Salvati, E. Guidelines for use of the flexible fiberoptic sigmoidoscope in the management of the surgical patient. Dis Colon Rectum 1982, 25: $187-190$

13. Vellacott, K.D., Amar, S.S. \& Hardcastle, J.D. Comparison of rigid and flexible fibreoptic sigmoidoscopy with double contrast barium enemas. Br J Surg 1982, 69: 399-400.

14. Thoeni, R.F. \& Petras, A. Detection of rectal and rectosigmoid lesions by double-contrast barium enema examination and sigmoidoscopy. Diag Rad 1982, 142: 59-62.

15. Knudsen, J.T., Moesgaard, F. \& Pedersen, T. The value of conventional barium enema of the colon in patients with haemorrhoids. Acta Chir Scand 1982, 148: 87-88.

16. Wherry, D.C. Screening for colorectal neoplasia in asymptomatic patients using flexible fiberoptic sigmoidoscopy. Dis Colon Rectum 1981, 24: 521-522.

17. Gilbertsen, V.A. Proctosigmoidoscopy and polypectomy in reducing the incidence of rectal cancer. Cancer 1974 supplement, 34: 936-939.

18. Spencer, R.J., Melton, L.J., Ready, R.L. \& Ilstrup, D.M. Treatment of small colorectal polyps: a population-based study of the risk of subsequent carcinoma. Mayo Clin Proc 1984, 59: 305-310.

19. Muto, T., Bussey, H.J.R. \& Morson, B.C. The evolution of cancer of the colon and rectum. Cancer 1975, 36: 2251-2270. 